\title{
Image Restoration using a Nonlinear Second-order Parabolic PDE-based Scheme
}

\author{
Tudor Barbu and Costică Moroşanu
}

\begin{abstract}
A novel anisotropic diffusion-based image denoising and restoration approach is proposed in this paper. A variational model for image restoration is introduced first, then the corresponding Euler-Lagrange equation being determined. A nonlinear parabolic PDE model is then obtained from this equation. It is based on a novel edge-stopping function and conductance parameter. A serious mathematical treatment is performed on this second-order anisotropic diffusion scheme, its wellpossedness being investigated. Then, a consistent explicit numerical approximation scheme based on the finite difference method is developed for the proposed PDE model.
\end{abstract}

\section{Introduction}

Because of their feature-preserving advantage, the nonlinear second-order diffusion based techniques represent the most effective image enhancement tool [1]. They have been widely used in the image denoising and restoration domain since 1987, when the influential anisotropic diffusion algorithm of Perona and Malik was introduced [2]. Numerous anisotropic diffusion models for image denoising derived from Perona-Malik scheme have been proposed since then [3]

Key Words: image denoising and restoration, qualitative properties of solutions, boundary value problems for nonlinear parabolic PDE, Leray-Schauder principle, edge-stopping function, finite differences, numerical approximation scheme.

2010 Mathematics Subject Classification: Primary 35Bxx, 94A08; Secondary 35K55, 35K60, 35Qxx, 65Nxx, 68U10

Received: February, 2016.

Revised: March, 2016.

Accepted: April, 2016. 
Also, since it is common to obtain PDE (partial differential equation) models from variational schemes, many variational restoration approaches have been also developed in the last 25 years $[1,3]$. The most influential one is the Total Variation (TV) Denoising proposed by Rudin, Osher and Fetami in 1992 [4].

Because the conventional two-dimension filters and most linear PDE-based smoothing methods suffer from blurring effect that destroys the edges and other important image details, and also do not have the localization property, the detail-preserving restoration still constitutes a serious challenge for the image processing researchers. Although the nonlinear second-order PDE overcome these drawbacks of the classic 2D filters, avoiding the image blurring, preserving the edges very well and having the localization property, they are often affected by another undesired effect, namely the staircase, or blocky, effect [5].

Many second-order nonlinear diffusion based restoration techniques that reduce this effect has been proposed in the last years. We could mention here several improved versions of the Perona-Malik algorithm and TV denoising, such as the Weickert diffusion [3], Adaptive TV denoising [6], anisotropic HDTV regularizer [7] and TV Denoising with Split Bregman [8]. We also developed such nonlinear second-order PDE and variational denosing models that overcome the staircasing and other unintended effects, in our previous papers [9-12]. A more effective second-order nonlinear anisotropic diffusionbased feature-preserving restoration technique is provided in this article. It provides a satisfactory image noise removal while reducing considerably the blurring and staircase effects.

Our PDE variational model for image restoration is described in the next section. A nonlinear diffusion scheme is obtained from the variational problem by applying the corresponding Euler-Lagrange equation. Then, a mathematical treatment of the proposed denoising model, investigating its well-posedness and the selection of the edge-stopping function, is provided in the third section. A consistent numerical approximation scheme developed for this model is described in the fourth section, while the image restoration experiments performed by using this filtering technique are discussed in the fifth section. The article ends with a conclusions section and a list of references.

\section{Novel Anisotropic Diffusion Model for Image Restoration}

A new effective PDE-based denoising and restoration technique is described in this section. As we have already mentioned, it is intended to optimize the trade-off between the image filtering, detail preservation and unintended effect removal.

First, we consider the following variational problem [13] that minimizes an 
energy cost functional:

$$
u_{\text {optim }}=\arg \min _{u} E(u),
$$

where the result $u_{\text {optim }}$ would represent the enhanced (restored) image and

$$
E(u)=\int_{\Omega}\left(\frac{\lambda}{2} \psi_{u}(\|\nabla u\|)+\frac{\rho}{2}\left(u-u_{0}\right)^{2}\right) d \Omega
$$

where the image domain $\Omega \subseteq R^{2}$, the parameters $\lambda, \rho \in(0,1)$ and $u_{0}$ is the initial image, affected by Gaussian noise. The regularizer function of this energy functional has the following form:

$$
\psi_{u}(s)=\int_{0}^{s} \tau \zeta\left(\frac{\gamma(u)}{\beta \ln (s+\gamma(u))^{3}+\delta}\right)^{1 / 2} d \tau
$$

its conductance parameter being modeled as a function of some statistics of the evolving image, as following:

$$
\gamma(u)=\alpha \cdot \mu(\|\nabla u\|)+\eta \cdot \operatorname{pos}(u)
$$

where $\alpha, \beta, \zeta, \eta, \delta \in(0,3], \mu$ returns the average value and pos $(u)$ gets the position of $u$ in the evolving sequence.

Let us denote $L\left(x, y, u, u_{x}, u_{y}\right)=\frac{\lambda \psi_{u}(\|\nabla u\|)+\rho\left(u-u_{0}\right)^{2}}{2}$, where $u_{x}=\partial u / \partial x$, then determine the Euler-Lagrange equation corresponding to the proposed variational scheme [13]. The respective equation is obtained as:

$$
\frac{\partial L}{\partial u}-\frac{\partial}{\partial x} \frac{\partial L}{\partial u_{x}}-\frac{\partial}{\partial y} \frac{\partial L}{\partial u_{y}}=0
$$

that leads to

$$
\rho\left(u-u_{0}\right)-\frac{\partial}{\partial x}\left(\frac{\lambda}{2} \psi_{u}^{\prime}(\|\nabla u\|) \frac{2 u_{x}}{\|\nabla u\|}\right)-\frac{\partial}{\partial y}\left(\frac{\lambda}{2} \psi_{u}^{\prime}(\|\nabla u\|) \frac{2 u_{y}}{\|\nabla u\|}\right)=0
$$

which is equivalent to

$$
\rho\left(u-u_{0}\right)-\lambda \operatorname{div}\left(\frac{\psi_{u}^{\prime}(\|\nabla u\|)}{\|\nabla u\|} \nabla u\right)=0 .
$$

If we note $\xi_{u}(s)=\frac{\psi^{\prime}(s)}{s}$, then (7) becomes:

$$
\rho\left(u-u_{0}\right)-\lambda \operatorname{div}\left(\xi_{u}(\|\nabla u\|) \nabla u\right)=0 .
$$


One then applies the steepest descent method [13] on this PDE and, by applying some boundary conditions, obtains the following nonlinear anisotropic diffusion model:

$$
\left\{\begin{array}{l}
\frac{\partial u}{\partial t}=\lambda \operatorname{div}\left(\xi_{u}(\|\nabla u\|) \nabla u\right)-\rho \cdot\left(u-u_{0}\right) \\
u(0, x, y)=u_{0} \\
u(t, x, y)=0, \quad \forall t \geq 0,(x, y) \in \partial \Omega
\end{array},(x, y) \in \Omega .\right.
$$

Obviously, the edge-stopping (diffusivity) function of this parabolic PDEbased denoising scheme is $\xi_{u}:[0, \infty) \rightarrow(0, \infty)$, which is determined, from equation (3), as following:

$$
\xi_{u}(s)=\frac{\psi_{u}^{\prime}(s)}{s}=\zeta \sqrt{\frac{\gamma(u)}{\beta \ln (s+\gamma(u))^{3}+\delta}} .
$$

The minimization result given by (1), which represents the restored image, is then computed by solving the PDE (9). The existence and uniqueness of the solution of this parabolic diffusion-based model are discussed in the next section, where a mathematical treatment is performed for it.

\section{A Mathematical Treatment of the PDE-based Model}

In this section we perform a mathematical investigation of the proposed variational PDE model. Thus, the obtained diffusivity (edge-stopping) function is analyzed first, then the well-posedness of the anisotropic diffusion model.

So, the function $\xi_{u}$ is always positive, because $\psi_{u}(s)>0, \forall s \geq 0$. Also, it represents a monotonically decreasing function, because $\forall s_{1} \leq s_{2}$,

$$
\xi_{u}\left(s_{1}\right)=\zeta \sqrt{\frac{\gamma(u)}{\beta \ln (s+\gamma(u))^{3}+\delta}} \geq \zeta \sqrt{\frac{\gamma(u)}{\beta \ln (s+\gamma(u))^{3}+\delta}}=\xi_{u}\left(s_{2}\right) .
$$

This edge-stopping function is also convergent to zero, since we have: $\lim _{s \rightarrow \infty} \xi_{u}(s)=\lim _{s \rightarrow \infty} \zeta \sqrt{\frac{\gamma(u)}{\beta \ln (s+\gamma(u))^{3}+\delta}}=0$. Because it satisfies these conditions, the edge-stopping function $\xi_{u}$ is appropriate for an effective denoising.

In the following we will describe the framework of our problem (9), namely, on a bounded domain $\Omega \subset \mathbb{R}^{2}$, with a $C^{2}$ boundary $\partial \Omega$ and for a finite time 
$T>0$, we consider the following second boundary value problem

$$
\begin{cases}\frac{\partial}{\partial t} u\left(t, x_{1}, x_{2}\right)=\lambda \operatorname{div}\left(\xi_{u}(\|\nabla u\|) \nabla u\right) & \\ \quad-\rho\left(u\left(t, x_{1}, x_{2}\right)-u_{0}\left(x_{1}, x_{2}\right)\right)+f\left(t, x_{1}, x_{2}\right) & \text { in } Q=(0, T] \times \Omega \\ \lambda \xi_{u}(\|\nabla u\|) \frac{\partial}{\partial \nu} u\left(t, x_{1}, x_{2}\right)=0 & \text { on } \Sigma=(0, T] \times \partial \Omega \\ u\left(0, x_{1}, x_{2}\right)=u_{0}\left(x_{1}, x_{2}\right) \in W_{\infty}^{2-\frac{2}{p}}(\Omega) & \text { on } \Omega,\end{cases}
$$

where $\lambda, \rho$ are positive constants, $\xi_{u_{0}}\left(\left\|\nabla u_{0}\right\|\right) \frac{\partial}{\partial \nu} u_{0}\left(x_{1}, x_{2}\right)=0$ and $p \geq 2$.

Basic tools in our approach are the Leray-Schauder degree theory and the $L^{p}$-theory of linear and quasi-linear parabolic equations [14]. In the following, for a given positive integer $k$ and $1 \leq p \leq \infty, W_{p}^{k, 2 k}(Q)$ denote the Sobolev space on $Q$ :

$$
W_{p}^{k, 2 k}(Q)=\left\{y \in L^{p}(Q): \frac{\partial^{r}}{\partial t^{r}} \frac{\partial^{q}}{\partial x^{q}} y \in L^{p}(Q), \text { for } 2 r+q \leq k\right\},
$$

i.e., the spaces of functions whose $t$-derivatives and $x$-derivatives up to the order $k$ and $2 k$, respectively, belong to $L^{p}(Q)$ (see [14, p. 5]).

Also, we shall use the set $C^{1,2}(\bar{Q})\left(C^{1,2}(Q)\right)$ of all continuous functions in $\bar{Q}$ (in $Q$ ) having continuous derivatives $u_{t}, u_{x}, u_{x x}$ in $\bar{Q}$ (in $Q$ ), as well as the Sobolev spaces $W_{\infty}^{2-2 / p}(\Omega), W_{p}^{l, l / 2}(\Sigma)$ with non integral $l$ for the initial and boundary conditions, respectively (see [14, p. 8, p. 70 and p. 81]).

Definition 1. The function $u(t, x), x=\left(x_{1}, x_{2}\right)$ is a classical solution of the second boundary value problem (12) if it is continuous in $\bar{Q}$, have continuous derivatives $u_{t}, u_{x}, u_{x x}$ in $Q$, satisfy the equation $(12)_{1}$ at all points $(t, x) \in Q$ and satisfy conditions $(12)_{2}$ and $(12)_{3}$ for $(t, x) \in \Sigma$ and $t=0$, respectively.

For convenience, we will write problem (12) in the equivalent form

$$
\begin{cases}\frac{\partial}{\partial t} u\left(t, x_{1}, x_{2}\right)-\lambda \frac{\partial}{\partial u_{x_{j}}}\left(\xi_{u}(\|\nabla u\|) u_{x_{i}}\right) u_{x_{j} x_{i}} & \\ \quad+A\left(t, x_{1}, x_{2}, u, u_{x_{i}}\right)=\rho u_{0}\left(x_{1}, x_{2}\right)+f\left(t, x_{1}, x_{2}\right) & \text { in } Q \\ \lambda \xi_{u}(\|\nabla u\|) \frac{\partial}{\partial \nu} u\left(t, x_{1}, x_{2}\right)=0 & \text { on } \Sigma \\ u\left(0, x_{1}, x_{2}\right)=u_{0}\left(x_{1}, x_{2}\right) & \text { on } \Omega,\end{cases}
$$

with

$$
\begin{gathered}
A\left(t, x_{1}, x_{2}, u, u_{x_{i}}\right)=-\lambda \frac{\partial}{\partial u}\left(\xi_{u}(\|\nabla u\|) u_{x_{i}}\right) u_{x_{i}} \\
-\lambda \frac{\partial}{\partial x_{i}}\left(\xi_{u}(\|\nabla u\|) u_{x_{i}}\right)+\rho u\left(t, x_{1}, x_{2}\right)
\end{gathered}
$$


and

$$
u_{x_{i}}=\frac{\partial}{\partial x_{i}} u\left(t, x_{1}, x_{2}\right), \quad u_{x_{j} x_{i}}=\frac{\partial^{2}}{\partial x_{j} \partial x_{i}} u\left(t, x_{1}, x_{2}\right), \quad i, j=1,2 .
$$

In the present work we will investigate the solvability of the second boundary value problems of the form (13) in the class $W_{p}^{1,2}(Q)$. Thereat our main result in studying the existence and regularity of solution in problem (13) is the following

Theorem 1. Suppose $u\left(t, x_{1}, x_{2}\right) \in C^{1,2}(Q)$ is a classical solution of equation (13) satisfying $\lambda \xi_{u}(\|\nabla u\|) \frac{\partial}{\partial \nu} u\left(t, x_{1}, x_{2}\right)=0$ on the lateral surface $\Sigma$ of the cylinder $Q$ and that the following conditions hold

$I_{1}$. For $\left(t, x_{1}, x_{2}\right) \in Q,\left|u\left(t, x_{1}, x_{2}\right)\right|<M, M>0$, and arbitrary $p$ the function $\xi_{u}(\|\nabla u\|) p$ are continuous, differentiable with respect to $x=\left(x_{1}, x_{2}\right), u$ and $p$ and satisfies the inequalities $\left(\xi=\left(\xi_{1}, \cdots, \xi_{n}\right)\right.$ an arbitrary real vector)

$$
\begin{gathered}
\nu \xi^{2} \leq \frac{\partial}{\partial p_{j}}\left(\xi_{u}(\|\nabla u\|) p\right) \xi_{i} \xi_{j} \leq \mu \xi^{2}, \quad \nu>0 ; \\
{\left[\left|\xi_{u}(\|\nabla u\|) u_{x_{i}}\right|+\left|\frac{\partial}{\partial u}\left(\xi_{u}(\|\nabla u\|) u_{x_{i}}\right)\right|\right](1+|p|)} \\
+\left|\frac{\partial}{\partial x_{1}}\left(\xi_{u}(\|\nabla u\|) u_{x_{1}}\right)\right|+\left|\frac{\partial}{\partial x_{2}}\left(\xi_{u}(\|\nabla u\|) u_{x_{1}}\right)\right| \\
+\left|\frac{\partial}{\partial x_{1}}\left(\xi_{u}(\|\nabla u\|) u_{x_{2}}\right)\right|+\left|\frac{\partial}{\partial x_{2}}\left(\xi_{u}(\|\nabla u\|)\right) u_{x_{2}}\right|+\left|u\left(t, x_{1}, x_{2}\right)\right| \leq \mu(1+|p|)^{2} ;
\end{gathered}
$$

$I_{2} \cdot\left\|\xi_{u}(\|\nabla u\|) u_{x_{i}}\right\|_{L^{r}(Q)}<M_{1}, \quad i=1,2, \quad\|u\|_{L^{s}(Q)} \leq M_{2}, \quad M_{1}, M_{2}>0$, with

$$
r=\left\{\begin{array}{ll}
\max \{p, 4\} & p \neq 4 \\
4+\varepsilon & p=4,
\end{array} ; \quad s= \begin{cases}\max \{p, 2\} & p \neq 2 \\
2+\varepsilon & p=2\end{cases}\right.
$$

and $\varepsilon$ being an arbitrarily small positive number.

Then, for any $f \in L^{p}(Q)$ and $u_{0} \in W_{\infty}^{2-\frac{2}{p}}(\Omega)$, with $p \neq \frac{3}{2}$, problem (13) has a solution $u \in W_{p}^{1,2}(Q)$ and satisfies the estimate

$$
\|u\|_{W_{p}^{1,2}(Q)} \leq C\left(\left\|u_{0}\right\|_{W_{\infty}^{2-\frac{2}{p}}(\Omega)}+\|f\|_{L^{p}(Q)}\right),
$$

where the constant $C>0$ is independent of $u$ and $f$. 
Proof. In order to prove the results on problem (13), we will apply the Leraly-Schauder principle. In this line, we will choose as suitable Banach space $B=W_{p}^{0,1}(Q)$, endowed with the norm

$$
\|v\|_{B}=\|v\|_{L^{p}(Q)}+\left\|v_{x}\right\|_{L^{p}(Q)}
$$

and we introduce the nonlinear operator

$$
u=u(v, \tau)=\Phi(v, \tau), \quad \forall(v, \tau) \in W_{p}^{0,1}(Q) \times[0,1],
$$

where $u(v, \tau)$ is the unique solution to the following linear boundary value problem

$$
\begin{cases}\frac{\partial}{\partial t} u\left(t, x_{1}, x_{2}\right)-\lambda\left[\tau \frac{\partial}{\partial v_{x_{j}}}\left(\xi_{v}(\|\nabla u\|) v_{x_{i}}\right)+(1-\tau) \delta_{i}^{j}\right] u_{x_{i} x_{j}} & \\ \quad=-\tau\left[A\left(t, x_{1}, x_{2}, v, v_{x_{i}}\right)-\rho u_{0}\left(x_{1}, x_{2}\right)+f\left(t, x_{1}, x_{2}\right)\right] & \text { in } Q \\ \lambda \xi_{v}(\|\nabla u\|) \frac{\partial}{\partial \nu} u\left(t, x_{1}, x_{2}\right)=0 & \text { on } \Sigma \\ u\left(0, x_{1}, x_{2}\right)=\tau u_{0}\left(x_{1}, x_{2}\right), & \text { on } \Omega .\end{cases}
$$

The nonlinear operator $\Phi$ in (18) depends on $\tau \in[0,1]$ and its fixed point for $\tau=1$ are solutions of problem (13).

$\Phi$ is well-defined (problem (19) has a solution). From the right-hand side of (19), it follows that $\forall v \in W_{p}^{0,1}(Q)$, then $A\left(t, x_{1}, x_{2}, v, v_{x_{i}}\right)+f\left(t, x_{1}, x_{2}\right) \in$ $L^{p}(Q)$. Using $L^{p}$-theory of linear parabolic equations (see [14, p. 341-342]), the solution $u$ to problem (19) exists and is unique with

$$
u=u(v, \tau) \in W_{p}^{1,2}(Q), \quad \forall v \in W_{p}^{0,1}(Q), \quad \forall \tau \in[0,1] .
$$

We have the continuous inclusions (see [15, p. 24])

$$
W_{p}^{1,2}(Q) \subset W_{p}^{0,1}(Q),
$$

which means that $\Phi(v, \tau)=u \in W_{p}^{0,1}(Q)$ for all $v \in W_{p}^{0,1}(Q)$ and $\forall \tau \in[0,1]$. $\Phi$ is continuous and compact. Let $v^{n} \rightarrow v$ in $W_{p}^{0,1}(Q)$ and $\tau_{n} \rightarrow \tau$ in $[0,1]$. Denote

$$
u^{n, \tau_{n}}=\Phi\left(v^{n}, \tau_{n}\right), u^{n, \tau}=\Phi\left(v^{n}, \tau\right) \text { and } u^{\tau}=\Phi(v, \tau) .
$$


Then $\Phi\left(v^{n}, \tau_{n}\right)-\Phi\left(v^{n}, \tau\right)$ gives (see (18) and (19))

$$
\left\{\begin{array}{cc}
\frac{\partial}{\partial t}\left(u^{n, \tau_{n}}-u^{n, \tau}\right)-\lambda\left[\tau \frac{\partial}{\partial v_{x_{j}}^{n}}\left(\xi_{v^{n}}\left(\| \nabla v^{n} \mid\right) v_{x_{i}}^{n}\right)+(1-\tau) \delta_{i}^{j}\right]\left(u_{x_{i} x_{j}}^{n, \tau_{n}}-u_{x_{i} x_{j}}^{n, \tau}\right) & \\
\quad=-\left(\tau_{n}-\tau\right)\left\{\lambda\left[\frac{\partial}{\partial v_{x_{j}}^{n}}\left(\xi_{v^{n}}\left(\left\|\nabla v^{n}\right\|\right) v_{x_{i}}^{n}\right)-\delta_{i}^{j}\right] u_{x_{i} x_{j}}^{n, \tau_{n}}\right. & \\
\left.\quad+A\left(t, x, v^{n}, v_{x_{i}}^{n}\right)-\rho u_{0}\left(x_{1}, x_{2}\right)+f\left(t, x_{1}, x_{2}\right)\right\} & \text { in } Q \\
\lambda \xi_{v^{n}}(\|\nabla u\|) \frac{\partial}{\partial \nu}\left(u^{n, \tau_{n}}-u^{n, \tau}\right)=0 & \text { on } \Sigma \\
\left(u^{n, \tau_{n}}-u^{n, \tau}\right)\left(0, x_{1}, x_{2}\right)=\left(\tau_{n}-\tau\right) u_{0}\left(x_{1}, x_{2}\right) & \text { in } \Omega .
\end{array}\right.
$$

Owing that $u^{n, \tau_{n}} \in W_{p}^{1,2}(Q)$, the right-hand side of (22) belongs to $L^{p}(Q)$. Thus we may apply $L^{p}$-theory to problem (22) which gives the estimate

$$
\begin{aligned}
\left\|u^{n, \tau_{n}}-u^{n, \tau}\right\|_{W_{p}^{1,2}(Q)} \leq C\left|\tau_{n}-\tau\right|\left[\left\|\left(\frac{\partial}{\partial v_{x_{j}}^{n}}\left(\xi_{v^{n}}\left(\left\|\nabla v^{n}\right\|\right) v_{x_{i}}^{n}\right)-\delta_{i}^{j}\right) u_{x_{i} x_{j}}^{n, \tau_{n}}\right\|_{L^{p}(Q)}\right. \\
\left.+\left\|A\left(t, x, v^{n}, v_{x_{i}}^{n}\right)\right\|_{L^{p}(Q)}+\left\|u_{0}\right\|_{W_{\infty}^{2-\frac{2}{p}(\Omega)}}+\|f\|_{L^{p}(Q)}\right],
\end{aligned}
$$

for a constant $C\left(|\Omega|, p, \lambda, \rho, \mu, M, M_{1}\right)$. By virtue of (15) and $\mathrm{I}_{2}$ and, knowing that $u_{x_{i} x_{j}}^{n, \tau_{n}} \in L^{p}(Q)$, we derive the boundedness of the terms $A\left(t, x, v^{n}, v_{x_{i}}^{n}\right)$, $\left(\frac{\partial}{\partial v_{x_{j}}^{n}}\left(\xi_{v^{n}}\left(\left\|\nabla v^{n}\right\|\right) v_{x_{i}}^{n}\right)-\delta_{i}^{j}\right) u_{x_{i} x_{j}}^{n, \tau_{n}}, \quad u_{0}$ and $f$ in $L^{p}(Q)$. Thus, in view of the convergence $\tau_{n} \rightarrow \tau$, from the above inequality we get

$$
\left\|u^{n, \tau_{n}}-u^{n, \tau}\right\|_{W_{p}^{1,2}(Q)} \rightarrow 0 \text { for } n \rightarrow \infty .
$$

From (18) and (19) we also obtain: $\Phi\left(v^{n}, \tau\right)-\Phi(v, \tau)$

$$
\left\{\begin{aligned}
\frac{\partial}{\partial t}\left(u^{n, \tau}-u^{\tau}\right)-\lambda\left[\tau \frac{\partial}{\partial v_{x_{j}}^{n}}\left(\xi_{v^{n}}\left(\| \nabla v^{n} \mid\right) v_{x_{i}}^{n}\right)+(1-\tau) \delta_{i}^{j}\right]\left(u_{x_{\tau} x_{j}}^{n, \tau}-u_{x_{i} x_{j}}^{\tau}\right) & \\
\left.=-\tau\left[\lambda \frac{\partial}{\partial v_{x_{j}}^{n}}\left(\xi_{v^{n}}\left(\| \nabla v^{n}||\right) v_{x_{i}}^{n}\right)-\lambda \frac{\partial}{\partial v_{x_{j}}}\left(\xi_{v}\|\mid \nabla v\|\right) v_{x_{i}}\right)\right] u_{x_{i} x^{j}}^{\tau} & \\
-\tau\left[A\left(t, x, v^{n}, v_{x_{i}}^{n}-A\left(t, x, v, v_{x_{i}}\right)\right]\right. & \text { in } Q \\
\lambda \xi_{v^{n}}\left(\left\|\nabla v^{n}\right\|\right) \frac{\partial}{\partial \nu}\left(u^{n, \tau}-u^{\tau}\right)=0 & \text { on } \Sigma \\
\left(u^{n, \tau}-u^{\tau}\right)\left(0, x_{1}, x_{2}\right)=0 & \text { on } \Omega .
\end{aligned}\right.
$$


The $L^{p}$-theory applied to problem (24) gives us the estimate

$$
\begin{aligned}
& \left\|u^{n, \tau}-u^{\tau}\right\|_{W_{p}^{1,2}(Q)} \\
& \quad \leq C\left[\left\|\lambda\left(\frac{\partial}{\partial v_{x_{j}}^{n}}\left(\xi_{v^{n}}\left(\left\|\nabla v^{n}\right\|\right) v_{x_{i}}^{n}\right)-\frac{\partial}{\partial v_{x_{j}}}\left(\xi_{v}(\|\nabla v\|) v_{x_{i}}\right)\right) u_{x_{i} x_{j}}^{\tau}\right\|_{L^{p}(Q)}\right. \\
& \left.\quad+\left\|A\left(t, x, v^{n}, v_{x_{i}}^{n}\right)-A\left(t, x, v, v_{x_{i}}\right)\right\|_{L^{p}(Q)}\right]
\end{aligned}
$$

for a positive constant $C$. The boundedness of the terms in right-hand side of above inequality and the convergence $v^{n} \rightarrow v$ in $W_{p}^{0,1}(Q)$ allow us to conclude that

$$
\left\|u^{n, \tau}-u^{\tau}\right\|_{W_{p}^{1,2}(Q)} \rightarrow 0 \text { as } n \rightarrow \infty .
$$

Making use of relations (23) and (25) we derive the continuity of the nonlinear operator $\Phi$ defined in (18). Moreover, writing $\Phi$ as the composition

$$
W_{p}^{0,1}(Q) \times[0,1] \rightarrow W_{p}^{1,2}(Q) \hookrightarrow W_{p}^{0,1}(Q),
$$

the compactness of $\Phi$ immediately follows.

The regularity of the solutions. Now, we will establish the existence of a number $\delta>0$ such that

$$
(u, \tau) \in W_{p}^{0,1}(Q) \times[0,1] \text { with } u=\Phi(u, \tau) \Rightarrow\|u\|_{B}<\delta .
$$

The equality $u=\Phi(u, \tau)$ in relation (26) is equivalent to

$$
\begin{cases}\frac{\partial}{\partial t} u\left(t, x_{1}, x_{2}\right)-\lambda\left[\tau \frac{\partial}{\partial u_{x_{j}}}\left(\xi_{u}(\|\nabla u\|) u_{x_{i}}\right)+(1-\tau) \delta_{i}^{j}\right] u_{x_{i} x_{j}} & \\ \quad=-\tau\left[A\left(t, x_{1}, x_{2}, u, u_{x_{i}}\right)-\rho u_{0}\left(x_{1}, x_{2}\right)+f\left(y, x_{1}, x_{2}\right)\right] & \text { in } Q \\ \lambda \xi_{u}(\|\nabla u\|) \frac{\partial}{\partial \nu} u\left(t, x_{1}, x_{2}\right)=0 & \text { on } \Sigma \\ u\left(0, x_{1}, x_{2}\right)=\tau u_{0}\left(x_{1}, x_{2}\right) & \text { on } \Omega .\end{cases}
$$

Applying $L^{p}$-theory to problem (27) and using the assumptions expressed in (15) and $I_{2}$, we deduce that

$$
\|u\|_{W_{p}^{1,2}(Q)} \leq C\left(\left\|u_{0}\right\|_{W_{\infty}^{2-\frac{2}{p}}(\Omega)}+\|f\|_{L^{p}(Q)}\right),
$$

for a constant $C\left(|\Omega|, p, \lambda, \mu, \nu, \rho, M_{1}, M_{2}\right)>0$ with $p \neq \frac{3}{2}$. The continuous embedding $W_{p}^{1,2}(Q) \subset W_{p}^{0,1}(Q)$ together with above inequality ensures that

$$
\|u\|_{W_{p}^{0,1}(Q)} \leq C\|u\|_{W_{p}^{1,2}(Q)},
$$


which means that the claim in (26) holds true.

Denoting

$$
B_{\delta}:=\left\{u \in B:\|u\|_{B}<\delta\right\},
$$

relation (26) implies that $\Phi(u, \tau) \neq u, \forall u \in \partial B_{\delta}, \forall \tau \in[0,1]$, provided that $\delta>0$ is sufficiently large. Furthermore, following the same reasoning as in [16], we conclude that the problem (13) has a solution $u \in W_{p}^{1,2}(Q)$. Estimate (28) permit as to conclude that (16) is valid.

\section{Robust Numerical Approximation Approach}

Now we must approximate the weak solution of the second-order parabolic PDE, whose existence has been proved in the preview section. Thus, the proposed nonlinear diffusion model is discretized by using the finite-difference method [17]. A consistent numerical approximation scheme is developed for this continuous model.

So, we consider a space grid size of $h$ and a time step $\Delta t$. The space and time coordinates are quantized as following:

$$
x=i h, \quad y=j h, \quad t=n \Delta t, \quad \forall i \in\{0, . ., I\}, j \in\{0, . . J\}, n \in\{0, . ., N\} .
$$

Since we have the following relation:

$$
\operatorname{div}\left(\xi_{u}(\|\nabla u\|) \nabla u\right)=\xi_{u}(\|\nabla u\|) \Delta u+\nabla\left(\xi_{u}(\|\nabla u\|)\right) \cdot \nabla u,
$$

we perform a finite difference-based discretization for each component of this sum. So, the first part of this sum is approximated by using the discrete Laplacian operator. Thus, we compute $\xi_{i, j}^{n}=\xi_{u}\left(\left\|\nabla u_{i, j}^{n}\right\|\right) \Delta u_{i, j}^{n}$ for $n \in\{0, . ., N\}$, where

$$
\Delta u_{i, j}^{n}=\frac{u^{n}(i+h, j)+u^{n}(i-h, j)+u^{n}(i, j+h)+u^{n}(i, j-h)-4 u^{n}(i, j)}{h^{2}}
$$

and

$$
\xi_{u}\left(\left\|\nabla u_{i, j}^{n}\right\|\right)=\xi_{u}\left(\sqrt{\frac{\left(u_{i+h, j}^{n}-u_{i-h, j}^{n}\right)^{2}}{4 h^{2}}+\frac{\left(u_{i, j+h}^{n}-u_{i, j-h}^{n}\right)^{2}}{4 h^{2}}}\right)
$$

is computed by using (10).

The second part of the sum is computed as following:

$$
\begin{aligned}
& \nabla\left(\xi_{u}(\|\nabla u\|)\right) \cdot \nabla u \\
& =\left(\frac{\partial}{\partial x} \xi_{u}\left(\sqrt{\left(\frac{\partial u}{\partial x}\right)^{2}+\left(\frac{\partial u}{\partial y}\right)^{2}}\right), \frac{\partial}{\partial y} \xi_{u}\left(\sqrt{\left(\frac{\partial u}{\partial x}\right)^{2}+\left(\frac{\partial u}{\partial y}\right)^{2}}\right)\right)\left(\frac{\partial u}{\partial x}, \frac{\partial u}{\partial y}\right)
\end{aligned}
$$


that leads to

$$
\nabla\left(\xi_{u}(\|\nabla u\|)\right) \cdot \nabla u=\frac{\partial \xi_{u}}{\partial s}(\|\nabla u\|) \frac{\left(\frac{\partial u}{\partial x}\right)^{2} \frac{\partial^{2} u}{\partial x^{2}}+\frac{\partial u}{\partial x} \frac{\partial u}{\partial y} \frac{\partial^{2} u}{\partial x \partial y}+\left(\frac{\partial u}{\partial y}\right)^{2} \frac{\partial^{2} u}{\partial y^{2}}+\frac{\partial u}{\partial x} \frac{\partial u}{\partial y} \frac{\partial^{2} u}{\partial x \partial y}}{\sqrt{\left(\frac{\partial u}{\partial x}\right)^{2}+\left(\frac{\partial u}{\partial y}\right)^{2}}} .
$$

We could perform some approximations in (35). Since the second order derivatives do not vary too much, the next approximation is performed:

$\nabla\left(\xi_{u}(\|\nabla u\|)\right) \cdot \nabla u \approx \frac{\partial \xi_{u}}{\partial s}(\|\nabla u\|) \frac{\frac{\partial^{2} u}{\partial x \partial y}\left(\frac{\partial u}{\partial x}+\frac{\partial u}{\partial y}\right)^{2}}{\sqrt{\left(\frac{\partial u}{\partial x}\right)^{2}+\left(\frac{\partial u}{\partial y}\right)^{2}}} \approx \xi_{u}^{\prime}\left(\sqrt{u_{x}^{2}+u_{y}^{2}}\right) u_{x y}\left(u_{x}+u_{y}\right)$,

where $u_{x}=\partial u / \partial x, u_{y}=\partial u / \partial y$ and $u_{x y}=\partial^{2} u / \partial x \partial y$ are discretized by applying the finite difference method [17].

Therefore $\xi_{u}^{\prime}\left(\sqrt{u_{x}^{2}+u_{y}^{2}}\right) u_{x y}\left(u_{x}+u_{y}\right)$ gets the discretization

$$
\begin{aligned}
& \xi_{u}^{\prime}\left(\sqrt{\frac{\left(u_{i+h, j}^{n}-u_{i-h, j}^{n}\right)^{2}}{4 h^{2}}+\frac{\left(u_{i, j+h}^{n}-u_{i, j-h}^{n}\right)^{2}}{4 h^{2}}}\right) \\
& \cdot \frac{\left(u_{i+h, j+h}^{n}-u_{i+h, j-h}^{n}-u_{i-h, j+h}^{n}+u_{i-h, j-h}^{n}\right)\left(u_{i+h, j}^{n}-u_{i-h, j}^{n}+u_{i, j+h}^{n}-u_{i, j-h}^{n}\right)}{8 h^{3}},
\end{aligned}
$$

where $u_{i, j}^{n}=u^{n}(i, j)$.

Then, one applies the obtained discretization of $\operatorname{div}\left(\xi_{u}(\|\nabla u\|) \nabla u\right)$ and the finite-difference method on the PDE in (9) and gets the following implicit approximation:

$$
\begin{aligned}
& \frac{u_{i, j}^{n+\Delta t}-u_{i, j}^{n}}{\Delta t}=\lambda \xi_{u}\left(\sqrt{\frac{\left(u_{i+h, j}^{n}-u_{i-h, j}^{n}\right)^{2}}{4 h^{2}}+\frac{\left(u_{i, j+h}^{n}-u_{i, j-h}^{n}\right)^{2}}{4 h^{2}}}\right) \\
& \cdot \frac{u_{i+h, j}^{n}+u_{i-h, j}^{n}+u_{i, j+h}^{n}+u_{i, j-h}^{n}-4 u_{i, j}^{n}}{h^{2}} \\
& +\lambda \xi_{u}^{\prime}\left(\sqrt{\frac{\left(u_{i+h, j}^{n}-u_{i-h, j}^{n}\right)^{2}}{4 h^{2}}+\frac{\left(u_{i, j+h}^{n}-u_{i, j-h}^{n}\right)^{2}}{4 h^{2}}}\right) \\
& \cdot \frac{\left(u_{i+h, j+h}^{n}-u_{i+h, j-h}^{n}-u_{i-h, j+h}^{n}+u_{i-h, j-h}^{n}\right)\left(u_{i+h, j}^{n}-u_{i-h, j}^{n}+u_{i, j+h}^{n}-u_{i, j-h}^{n}\right)}{8 h^{3}} \\
& -\rho\left(u_{i, j}^{n}-u_{i, j}^{0}\right) .
\end{aligned}
$$

If one consider $h=1$ and $\Delta t=1$, the above discretization is transformed 
into the next explicit numerical approximation scheme:

$$
\begin{aligned}
u_{i, j}^{n+1} & =u_{i, j}^{n}(1-\rho)+\lambda \xi_{u}\left(\frac{\sqrt{\left(u_{i+1, j}^{n}-u_{i-1, j}^{n}\right)^{2}+\left(u_{i, j+1}^{n}-u_{i, j-1}^{n}\right)^{2}}}{2}\right) \\
& \cdot\left(u_{i+1, j}^{n}+u_{i-1, j}^{n}+u_{i, j+1}^{n}+u_{i, j-1}^{n}-4 u_{i, j}^{n}\right) \\
& +\lambda \xi_{u}^{\prime}\left(\frac{\sqrt{\left(u_{i+1, j}^{n}-u_{i-1, j}^{n}\right)^{2}+\left(u_{i, j+1}^{n}-u_{i, j-1}^{n}\right)^{2}}}{2}\right) \\
& \cdot \frac{\left(u_{i+1, j+1}^{n}-u_{i+1, j-1}^{n}-u_{i-1, j+1}^{n}+u_{i-1, j-1}^{n}\right)\left(u_{i+1, j}^{n}-u_{i-1, j}^{n}+u_{i, j+1}^{n}-u_{i, j-1}^{n}\right)}{8} \\
& +\rho u_{i, j}^{0} .
\end{aligned}
$$

The iterative approximation algorithm provided by (18) is applied on the evolving image, for each $n \in\{0, \ldots, N\}$, starting with $u^{0}=u_{0}$, which represents the initial noisy $[I \times J]$ image. The developed explicit numerical approximation scheme is consistent to the nonlinear second-order PDE model given by (1). This scheme converges fast to the approximation of its unique and weak solution, representing the optimal restoration, $u^{N+1}$, since the number of iterations, $N$, takes low values.

\section{Experiments and Method Comparison}

The nonlinear second-order diffusion-based filtering technique proposed here has been tested on hundreds images affected by Gaussian noise, satisfactory restoration results being achieved. We used some well-known digital image collections for our denoising experiments, such as the Volumes of the USC-SIPI database, by corrupting their original images with various amounts of Gaussian noise and then applying the iterative denoising algorithm on them. We have determined on a trial and error basis the following values of parameters that provide an optimal smoothing: $\lambda=1.2, \rho=0.3, \eta=0.2, \beta=0.7, \alpha=1.3$, $\delta=4, \zeta=0.5, N=12$.

The performed denoising tests show that our PDE-based scheme reduces considerably the noise, while preserving the image boundaries and other important features. Also, it avoids unintended effects, such as image blurring, blocky effect [5] or speckle noise, and executes quite fast, having a running time of less than $1 s$.

Method comparison have been also performed. The performance of this PDE filtering approach has been assessed by using some well-known performance measures, such as Peak Signal-to-Noise Ratio (PSNR), Norm of the 
Error (NE) measure and the Structural Similarity Image Metric (SSIM) [18]. The PSNR values provided by our technique and other filtering approaches are displayed in Table 1. One can see that our second-order diffusion-based scheme gets higher PSNR values than other image restoration models.

It outperforms the conventional two-dimension image filters, such as Average, Gaussian, Wiener and Median filters, since it overcomes the image blurring and preserves the edges. Also, it outperforms some influential nonlinear diffusion schemes, such as both versions of the Perona-Malik anisotropic diffusion model and its derived methods [2], and the variational TV Denoising [4]. Unlike these second-order PDE-based approaches, it alleviates the staircase effect and also converges much faster.

Table 1. PSNR values achieved by various denoising techniques

\begin{tabular}{|l|l|}
\hline Restoration approach & PSNR \\
\hline This $2^{\text {nd }}$-order PDE scheme & $27.33(\mathrm{~dB})$ \\
\hline Average Filter & $25.63(\mathrm{~dB})$ \\
\hline Gaussian 2D & $25.47(\mathrm{~dB})$ \\
\hline Median Filter & $26.48(\mathrm{~dB})$ \\
\hline Perona-Malik 1 & $26.85(\mathrm{~dB})$ \\
\hline Perona-Malik 2 & $26.81(\mathrm{~dB})$ \\
\hline Tv Denoising & $27.14(\mathrm{~dB})$ \\
\hline
\end{tabular}

Some restoration results provided by these techniques are displayed in Fig. 1. The original $[512 \times 512]$ Elaine image is depicted in (a). Its version corrupted with an amount of Gaussian noise given by $\mu=0.04$ and variance $=0.05$, is displayed in (b). The results produced by the $[3 \times 3] 2 \mathrm{D}$ filters, like $2 \mathrm{D}$ Gaussian filter, Average and Median, are displayed in (c) - (e). The denoising results produced by the PDE-based and variational methods are displayed in (f) - Perona-Malik 1, (g) - Perona-Malik 2, (h) - TV Denoising, (i) - our restoration technique. One can see that image (i), which is bounded by a blue rectangle, represents the best enhancement result.

\section{Conclusions}

We have described a novel second-order nonlinear PDE-based restoration approach in this paper. The nonlinear anisotropic diffusion model proposed here represents the major contribution of this work. It is obtained from a PDE 


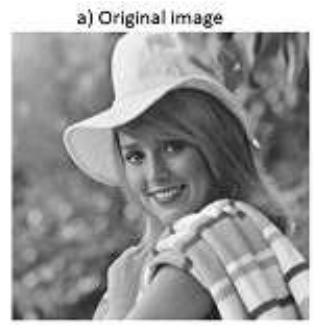

d) Gaussian 20

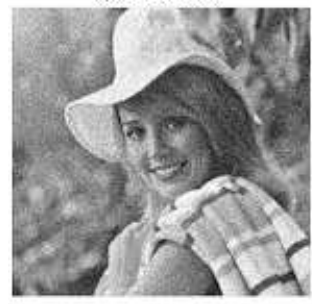

g) Perona-Malik 2

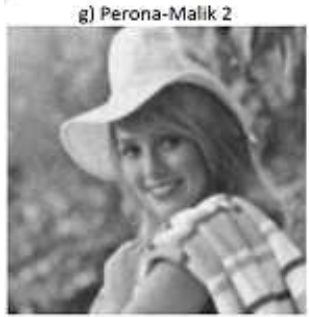

b) Noisy version

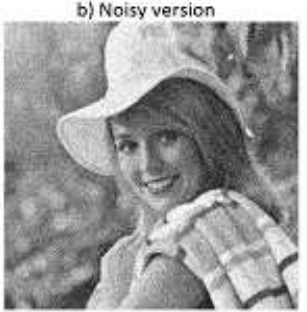

e) Median filtering

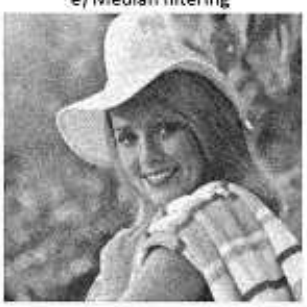

h) TV Denoising

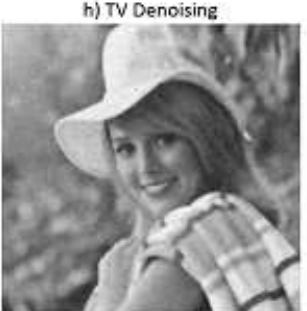

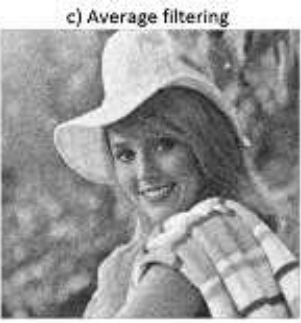

f) Perona-Malik 1

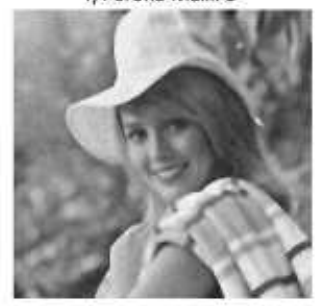

i) This diffusion model

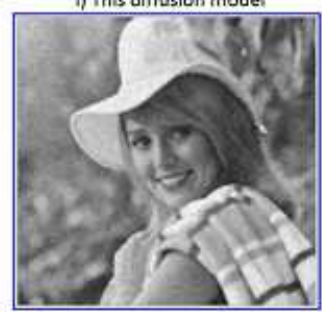

Figure 1: Restoration results provided by several denoising models

variational scheme that is based on a novel regularizer function, which leads to a robust edge-stopping function, properly constructed for an effective image denoising.

The rigorous mathematical investigation of the obtained second-order parabolic PDE model, which is performed here, represents another contribution of our article. We have mainly analyzed the well-posedness of the diffusion model, demonstrating the existence of a unique and weak solution of it.

Next, a consistent and explicit numerical approximation scheme is constructed for the proposed second-order PDE model. We use the finite-difference method to develop a fast-converging iterative discretization algorithm for the differential model.

Our denoising scheme provides an effective detail-preserving image restoration that is proved by our successful results and method comparison. It out- 
performs the two-dimension conventional filters, since it overcomes the image blurring. Also, it performs better than many state-of-the-art second-order nonlinear diffusion approaches, by executing faster and alleviating the staircase effect.

We will try to improve this diffusion-based restoration scheme, as part of our future research in this domain, by modelling new edge-stopping functions. Also we intend to further derive this nonlinear PDE model such that to obtain higher-order PDE denoising schemes, like the fourth-order PDE approaches, or hybrid smoothing models combining second-order and fourth-order PDEbased schemes.

\section{Acknowledgements}

This work was mainly supported by the project PN-II-RU-TE-2014-4-0083, financed by UEFSCDI Romania. It was supported also by the Institute of Computer Science of the Romanian Academy.

\section{References}

[1] F. Guichard, L. Moisan and J. M. Morel, "A review of P.D.E. models in image processing and image analysis", Journal de Physique, vol. 4, pp. 137-154, 2001.

[2] P. Perona, J. Malik, "Scale-space and edge detection using anisotropic diffusion", Proc. of IEEE Computer Society Workshop on Computer Vision, 16-22, nov. 1987.

[3] J. Weickert, Anisotropic Diffusion in Image Processing, European Consortium for Mathematics in Industry. B. G. Teubner, Stuttgart, Germany, 1998.

[4] L. Rudin, S. Osher and E. Fatemi, "Nonlinear total variation based noise removal algorithms", Physica D: Nonlinear Phenomena, 60, pp. 259-268, 1992.

[5] A. Buades, B. Coll, J. M. Morel, "The staircasing effect in neighborhood filters and its solution", IEEE Transactions on Image Processing, 15, 6, pp. 1499-1505, 2006.

[6] Q. Chen, P. Montesinos, Q. Sun, P. Heng, D. Xia, "Adaptive total variation denoising based on difference curvature", Image Vis. Comput., 28, pp. 298-306, 2010.

[7] Y. Hu, M. Jacob, "Higher degree total variation (HDTV) regularization for image recovery", IEEE Trans. Image Processing, 21, pp. 2559-2571, 2012. 
[8] J. F. Cai, S. Osher, Z. Shen, "Split Bregman methods and frame based image restoration", Multiscale Model. Sim., 8, Issue 2, pp. 337-369, 2009.

[9] T. Barbu, "Variational Image Denoising Approach with Diffusion Porous Media Flow", Abstract and Applied Analysis, Volume 2013.

[10] T. Barbu, "A Novel Variational PDE Technique for Image Denoising", Lecture Notes in Computer Science (Proc. of the $20^{\text {th }}$ International Conference on Neural Information Processing, ICONIP 2013, part III, Daegu, Korea, November 3-7, 2013), vol. 8228, pp. 501-508, Springer-Verlag Berlin Heidelberg, M. Lee et al. (Eds.), 2013.

[11] T. Barbu, A. Favini, "Rigorous mathematical investigation of a nonlinear anisotropic diffusion-based image restoration model", in Electronic Journal of Differential Equations, number 129, pp. 1-9, 2014.

[12] T. Barbu, "Robust anisotropic diffusion scheme for image noise removal", Procedia Computer Science (Proc. of $18^{\text {th }}$ International Conference in Knowledge Based and Intelligent Information \& Engineering Systems, KES 2014, Sept. 15-17, Gdynia, Poland), by Elsevier, 35, pp. 522-530, 2014.

[13] M. Hazewinkel, "Variational calculus", Encyclopedia of Mathematics, Springer, ISBN: 978-1-55608-010-4, 2001.

[14] O.A. Ladyzhenskaya, B.A. Solonnikov and N.N. Uraltzava, Linear and quasi-linear equations of parabolic type, Prov. Amer. Math. Soc., 1968.

[15] J.L. Lions, Control of distributed singular systems, Gauthier-Villars, Paris, 1985.

[16] C. Moroşanu, Analysis and optimal control of phase-field transition system: Fractional steps methods, Bentham Science Publishers, 2012, http://dx.doi.org/10.2174/97816080535061120101.

[17] P. Johnson, Finite Difference for PDEs, School of Mathematics, University of Manchester, Semester I, 2008.

[18] K. H. Thung, P. Raveendran, "A survey of image quality measures", Proc. International Conference for Technical Postgraduates (TECHPOS), pp. 1-4, 2009.

Tudor BARBU,

Institute of Computer Science of the Romanian Academy,

Iasi, Romania.

Email: tudor.barbu@iit.academiaromana-is.ro

Costică MOROŞANU,

Department of Mathematics,

Al. I. Cuza University of Iasi,

Bdul Carol I 11, 700506 Iasi, Romania.

Email: costica.morosanu@uaic.ro 